\title{
Tratamiento de efluentes textiles con luz ultravioleta solar
}

( ${ }^{1}$ ) Oscar Rafael Tinoco Gómez

$\left({ }^{2}\right)$ Ana María Medina Escudero

(3) Hernán Zapata Gamarra

\section{RESUMEN}

El uso de tecnologías limpias para el tratamiento de efluentes textiles es fundamental para alcanzar una contribución significativa, por parte de la industria textil, a un tratamiento ecológico de los mismos. Según la literatura revisada, los colorantes usados en la industria textil son difícilmente degradables y presentan fuerte oposición a tratamientos biológicos a que son sometidas las correspondientes aguas residuales.

El objetivo trazado con esta investigación, de carácter experimental, fue el de probar que la luz ultravioleta solar puede ser utilizada para el tratamiento de los efluentes textiles. El mismo que fue verificado, luego de realizar y analizar las pruebas de laboratorio correspondientes.

A menores concentraciones del peróxido de oxigeno (agua oxigenada) se logra una mejor pérdida de color del colorante. Se aprecia también que a menores concentraciones de dióxido de titanio la respuesta del sistema a la degradación del color, es mejor; ello podría explicarse porque a menores concentraciones de dióxido de titanio, la formación del radical hidroxilo es menor, aumentando así su eficiencia en el proceso. El tiempo óptimo de degradación del color es de $2 \mathrm{~h}$ y la concentración de dióxido de titanio para lograr dicho tiempo fue de $50 \mathrm{ppm}$.

Palabras clave: efluentes textiles, dióxido de titanio, luz ultravioleta, energía solar

Treatment OF EFFLUENT TEXTILES WITH ULTRAVIOLET LIGHT

\section{ABSTRACT}

The use of clean technologies for the treatment of textile effluents is essential to achieve a significant contribution by the textile industry, environmental treatment of them. According to the literature, the dyes used in textile industry are hardly degradable and have strong opposition to biological treatments are subjected to appropriate wastewater.

At lower concentrations of hydrogen peroxide (oxygenated water) leads to better dye fading. It also shows that lower concentrations of titanium dioxide system response to the degradation of color, is better, this could be because that lower concentrations of titanium dioxide, hydroxyl radical formation is lower, thus increasing its efficiency the process.

The optimum time for color degradation is $2 \mathrm{~h}$ and the concentration of titanium dioxide to achieve this time was $50 \mathrm{ppm}$.

Keywords: textile effluent, titanium dioxide, ultraviolet light, solar energy

\section{INTRODUCCIÓN}

El $59 \%$ del consumo total de agua en los países desarrollados se destina a uso industrial, el $30 \%$ a consumo agrícola y un $11 \%$ a gasto doméstico, según se constata en el primer informe de Naciones Unidas sobre el desarrollo de los recursos hídricos del mundo, Agua para todos, agua para la vida (marzo 2003). En el 2025, el consumo de agua destinada a uso industrial alcanzará los 1170 km³/año, cifra que en 1995 se situaba en 752 km³/año. El sector productivo no sólo es el que más gasta, también es el que más contamina.

La descarga de aguas residuales generadas en los procesos industriales contienen compuestos químicos: ácidos, bases, iones metálicos, entre otros, materia orgánica (en suspensión, dilución, etc.) que consumen el contenido de oxígeno del cuerpo receptor, imposibilitando de acuerdo a su concentración, la autopurificación de las aguas de dicho cuerpo a tal grado que puede interferir en su uso futuro.

La industria textil presenta uso intensivo del agua, tanto para la limpieza de la materia prima, como durante el teñido. El teñido es bastante sensible en este proceso, debido al uso de colorantes químicos, que se caracterizan por una elevada estabilidad frente a la temperatura, luz, ataques microbianos y detergentes.

Los colorantes usados en la industria textil son difícilmente degradables y presentan fuerte oposición a tratamientos biológicos a que son sometidas las correspondientes aguas residuales.

El objetivo trazado con esta investigación fue el de probar que la luz ultravioleta solar puede ser utilizada para el tratamiento de los efluentes textiles.

\section{ANTECEDENTES}

Juan Chacón [1] resalta que el tratamiento de aguas residuales con presencia de colorantes es un reto tecnológico que puede ser abordado mediante el uso de tratamientos avanzados de oxidación (TAO), "los mismos que han demostrado ser métodos prometedores para el tratamiento de agua contaminada con compuestos orgánicos no biodegradables".

1 Docente FII UNMSM. Maestría Marketing. Doctorado Medio Ambiente y Desarrollo Sostenible. E-mail: otinocog@gmail.com

2 Ing. Química. Docente FII UNMSM.

E-mail: anita_monina@yahoo.es

3 Ing. Químico. Docente TECSUP.

E-mail: hzapatagamarra@gmail.com 
Añade Chacón, que "entre los TAO se cuenta el foto-Fenton, el cual utiliza peróxido de hidrógeno y sales ferrosas para la generación de radicales $\mathrm{HO}$, así como radiación ultravioleta, radiación visible o una combinación de ambas. El radical hidroxilo tiene alto potencial redox, cercano a $2.80 \mathrm{~V}$ a $25^{\circ} \mathrm{C}$, inferior sólo al flúor. La radiación utilizada puede ser la energía solar, lo que representa otra ventaja adicional en cuanto a costos".

Garcés et al. [3] llevaron adelante una investigación sobre tratamiento de aguas residuales mediante fotocatálisis, en la ciudad colombiana de Antioquia, señalando que "el uso de esta tecnología permite degradar y mineralizar el colorante Naranja Reactivo 84 y puede abrir una ruta alternativa a los procesos tradicionales de oxidación.

Salas [7] desarrolló en la Facultad de Química e Ingenieria Química de la UNMSM un estudio experimental para el tratamiento de aguas residuales de la industria textil, mediante procesos físico-químicos conducentes a la coagulación y floculación de los residuos.

Silva et al. [8] desarrollaron en la Universidad Agraria de la Molina el sistema fotocatalítico UV/TiO2 sobre el azocolorante Azul - 71 del efluente líquido textil, mediante el uso de un espectrofotómetro.

\section{AGUAS RESIDUALES TEXTILES}

La industria textil es una industria que consume grandes cantidades de agua, energía y productos químicos auxiliares, además genera una gran cantidad de agua residual; estos efluentes poseen elevadas concentraciones de colorantes, contaminantes orgánicos refractarios, compuestos tóxicos, componentes inhibidores, tensoactivos, componentes clorados. Por lo tanto, se constituyen en uno de los efluentes de más difícil tratamiento.

Su actividad consiste en transformar fibras naturales (algodón, lana, seda, etc. ) o sintéticas (poliester, acrílicas, etc.) en prendas de vestir u otros productos.

Abarca a una gran cantidad de industrias, en muchos casos pequeñas o medianas, que desarrollan las diferentes partes del proceso productivo.

Las principales operaciones que desarrollan son:

a. Tratamiento de las materias primas (lavado, acondicionamiento, hilado).

b. Fabricación de los tejidos (diferentes métodos).

c. Acabado de los tejidos (cambiando propiedades físicas tales como el color). d. Fabricación de los productos finales (prendas de vestir, sábanas, cortinas, alfombras, etc.).

La contaminación de las aguas residuales (AR) textiles procede tanto de las impurezas o residuos presentes en la materia prima, como de los productos químicos añadidos durante su procesamiento. Las operaciones de tipo físico producen poca o ninguna agua residual pero las de tipo químico en vía húmeda sí generan un importante volumen.

Los principales componentes del agua residual son las impurezas naturales que se encuentran en las fibras naturales y los compuestos químicos agregados durante los procesos empleados para el tratamiento de fibra, hebras o tejidos. Las plantas de procesamiento textil utilizan una amplia variedad de tintes y otros compuestos químicos, incluidos los ácidos, bases, sales agentes humedecedores, tintes y otros acabados auxiliares. Muchos de estos no permanecen en el producto textil terminado, sino que se desechan después de un uso específico. El efluente combinado de una planta textil, por tanto, puede contener cualquiera de estos compuestos o todos ellos.

Figura N. ${ }^{0}$

\section{CLASIFICACIÓN DE LOS CONTAMINANTES EN LAS AGUAS RESIDUALES TEXTILES}

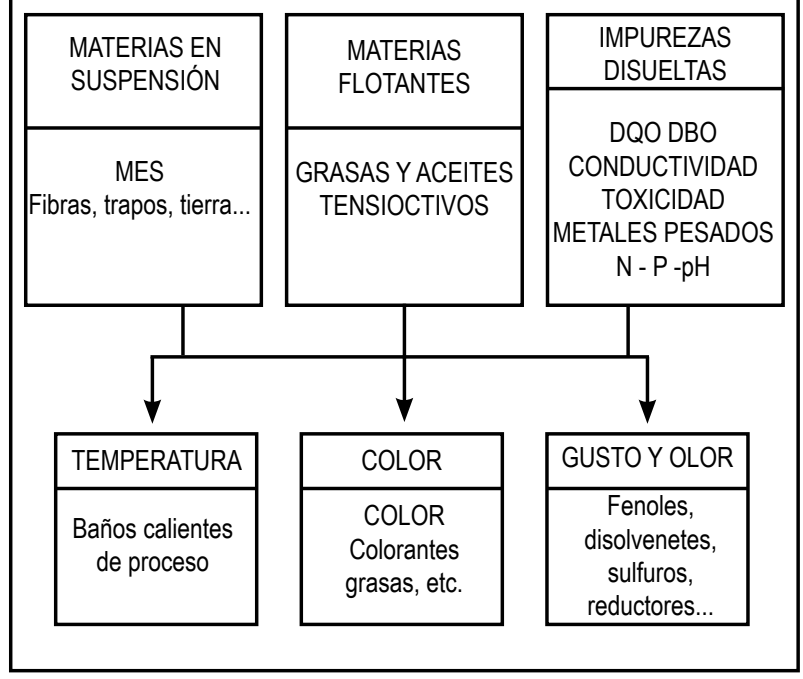

Fuente: Crespi, M. Boletín. INTEXTAR. 1987, NQ 92

Según Mansilla et al. [4], las corrientes de agua de descarga provienen principalmente del desengomado $(15 \%)$, descrude y mercerizado $(20 \%)$ y del blanqueo, teñido y lavado (65\%). El mayor aporte de la carga orgánica proviene de la etapa del desengomado que aporta alrededor de $50 \%$ del total del la DBO. 
La cantidad de agua empleada en los procesos textiles varía en forma considerable, dependiendo del proceso específico y del equipamiento utilizado por la planta. Por ejemplo, en el teñido con colorantes dispersos, se utilizan entre 100 y 150 litros de agua por kilogramo de producto. En el teñido con colorantes reactivos, las cifras varían entre 125 y 170 litros por kilogramo de producto.

Los procesos de la industria textil no liberan grandes cantidades de metales; sin embargo, aun las pequeñas concentraciones involucradas pueden producir acumulación en los tejidos de animales acuáticos. Muchas veces los efectos se observan a largo plazo, y en la mayoría de los casos son difíciles y costosos de tratar. Las descargas también pueden aumentar la población de peces y algas debido a su alto contenido de nitrógeno, y agotar en el largo plazo el contenido del oxígeno disuelto en el agua [4].

Los colorantes textiles tienen gran persistencia en el ambiente, y los métodos de eliminación clásicos no son útiles debido a que oxidaciones o reducciones parciales pueden generar productos secundarios altamente tóxicos.

Una gran proporción de los colorantes no son directamente tóxicos para los organismos vivos; sin embargo, la fuerte coloración que imparten a los medios de descarga puede llegar a suprimir los procesos fotosintéticos en los cursos de agua, por lo que su presencia debe ser controlada.

En general, las moléculas de los colorantes utilizados en la actualidad son de estructuras muy variadas y complejas. La mayoría de ellos son de origen sintético, muy solubles en agua, altamente resistentes a la acción de agentes químicos y poco biodegradables. Alrededor del $60 \%$ de los colorantes en uso en la industria textil actual son colorantes reactivos, que se caracterizan por formar una unión éter con la fibra, lo que garantiza una mayor duración del color en el tejido. Sus estructuras frecuentemente contienen grupos azo, antraquinona o ftalocianina.

La mayoría de los colorantes utilizados en la industria textil no son biodegradables, y por lo tanto, el tratamiento de sus residuos líquidos no se resuelve de manera sencilla.

\section{PROCESOS FOTOCATALÍTICOS (03/TIO2/UV Y H2O2/TIO2/UV)}

La oxidación fotocatalítica se basa en la fotoexcitación de un semiconductor sólido como resultado de la absorción de radiación electromagnética, en general en la zona del ultravioleta próximo. La radiación provoca la excitación de electrones en la banda de valencia del sólido, lo que origina la formación de huecos caracterizados por un potencial de oxidación muy elevado. En estos huecos no sólo se produce la oxidación de compuestos orgánicos adsorbidos, sino que es posible que tenga lugar la descomposición del agua para originar radicales hidroxilo que participan a su vez en las reacciones de degradación de la materia orgánica. El principal fotocatalizador es el dióxido de titanio, tanto en forma de rutilo como de anatasa. El dióxido de titanio puede ser activado mediante radicación ultravioleta hasta $380 \mathrm{~nm}$, lo que permite su funcionamiento como fotocatalizador solar puesto que la irradiación solar comienza a longitudes de onda de unos $300 \mathrm{~nm}$. Un $5 \%$ del total de la radiación solar podría aprovecharse de esta forma. La mayoría de los compuestos orgánicos contaminantes son susceptibles de ser tratados mediante fotocatálisis, incluyendo moléculas cloradas como clorofenoles y dioxinas, que resultan mineralizados hasta $\mathrm{CO}_{2}$ y $\mathrm{HCl}$. El pH influye en la reacción de fotooxidación de compuestos orgánicos, ya que no sólo condiciona el estado de la superficie, sino la adsorción de los contaminantes. Los mejores resultados se obtienen para valores de $\mathrm{pH}$ ligeramente ácidos y en combinación con otros generadores de hidroxilos, como el ozono o el peróxido de hidrógeno [6].

\section{ENERGÍA SOLAR}

Existe una aplicación de la energía solar que ha despertado el interés de investigadores, sobre todo a partir de la década de 1970. Se trata del empleo de la energía solar para la eliminación de contaminantes en aguas residuales mediante el uso de un fotocatalizador adecuado. Este sistema permite aprovechar directamente la energía solar que llega a la superficie terrestre para provocar una serie de reacciones químicas (redox) que dan lugar a la eliminación de los compuestos orgánicos en las aguas de vertidos urbanos, industriales y agrícolas, y cambiar el estado de oxidación de los metales pesados de tal forma que pasan de estar disueltos a una forma insoluble [8].

Una de las tecnologías de este tipo, que resulta atractiva para la descontaminación de aguas con sustancias orgánicas tóxicas, es la degradación fotocatalítica basada en el uso de dióxido de titanio $\left(\mathrm{TiO}_{2}\right)$ como fotocatalizador y luz ultravioleta solar de baja energía (320-390 nm) [8]. 


\section{ANÁLISIS Y DISCUSIÓN}

El trabajo de campo se realizó a partir de muestras extraídas de una planta de textiles ubicada en el cono norte de Lima y procesadas en laboratorio. Por razones de infraestructura, se simuló un efluente textil industrial en base a un colorante comercial, insumo entregado esta planta. La concentración fue de 100 ppm de rojo de synosol, acondicionada con hidróxido $4.0 \mathrm{M}$. El análisis fisicoquímico del efluente textil elaborado fue de $\mathrm{pH}=4.6$ y $5.6 \mathrm{NTU}$ (unidades nefelometrías) de turbidez. De acuerdo a la normativa nacional para desagües industriales, los valores de $\mathrm{pH}$ deben estar comprendidos entre 5 y 8.5 para que ingresen a la red pública. Pero durante el proceso de teñido, debido al uso de sustancias alcalinas para fijar el color, los efluentes con colorantes llegan a un pH entre 9 y 10. Por lo que, se debe bajar el $\mathrm{pH}$, para que sea descargado a la red pública. El valor de turbidez medido nos facilita el proceso de foto degradación del colorante. A mayores valores de turbidez más difícil sería la fotodegradación del colorante.

La muestra sintética de efluente con el colorante rojo de synosol se? acondiciona con solución de $\mathrm{NaOH}$ 4.0 M. Debido al acondicionamiento con hidróxido $4.0 \mathrm{M}$, la muestra textil pasa de un $\mathrm{pH}$ de 4.6 a uno de 6.0. Los experimentos de fotooxidación se iniciaron con este último $\mathrm{pH}$. Las muestras fueron leídas en un espectrofotómetro Genesys 10 UV a una ?longitud de onda de $525 \mathrm{~nm}$. La lámpara UV es una lámpara de mercurio de $8 \mathrm{~W}$.

Los materiales utilizados en las diferentes pruebas fueron el dióxido de titanio, peróxido de hidrógeno (agua oxigenada) y rojo de synozol.

La formación del agente oxidante es:

$$
\mathrm{H} 2 \mathrm{O} 2(\mathrm{a}) \longrightarrow 2 \mathrm{OH} .(\mathrm{ac})
$$

Cuadro 1. Valores termodinámicos de variación de entalpía $(\Delta H)$, de entropía $(\Delta S)$ y de energía libre de Gibbs $(\Delta G)$ de la reacción de formación del agente oxidante respecto a la

\begin{tabular}{|c|c|c|c|}
\hline \multicolumn{4}{|c|}{ temperatura. } \\
\hline $\mathrm{T}$ & deltaH & deltaS & deltaG \\
\hline $\mathrm{C}$ & kcal & $\mathrm{cal} / \mathrm{K}$ & kcal \\
\hline 0.000 & 43.525 & $-2.65 !$ & 44.249 \\
\hline 5.000 & 43.487 & -2.789 & 44.263 \\
\hline 10.000 & 43.445 & 2.938 & 44.277 \\
\hline 15.000 & 43.400 & -3.095 & 44.292 \\
\hline 20.000 & 43.353 & -3.258 & 44.308 \\
\hline 25.000 & 43.304 & -3.424 & 44.325 \\
\hline 30.000 & 43.253 & -3.593 & 44.342 \\
\hline 35.000 & $43.20 !$ & -3.764 & $44.36 !$ \\
\hline 40.000 & 43.148 & .3 .936 & 44.380 \\
\hline 45.000 & 43.093 & -4.108 & 44.400 \\
\hline 50.000 & 43.038 & .4 .281 & $44.42 !$ \\
\hline 55.000 & 42.982 & -4.453 & 44.443 \\
\hline 60.000 & $\$ 2.925$ & .4 .626 & $\$ 4.466$ \\
\hline 65.000 & 42.867 & -4.798 & 44.489 \\
\hline 70.000 & $\$ 2.808$ & .4970 & 44.514 \\
\hline 75.000 & 42.749 & .5 .142 & 44.539 \\
\hline 80.000 & $\$ 2.689$ & .5 .312 & 44.565 \\
\hline 85.000 & 42.628 & .5 .483 & 44.592 \\
\hline 90.000 & 42.567 & .5 .653 & $\$ 4.620$ \\
\hline 95.000 & 42.505 & .5 .822 & 44.649 \\
\hline 100.000 & 42.443 & $.599 !$ & 44.678 \\
\hline
\end{tabular}

Fuente: Software Termodinámico Programa HSC 6.0. Fabricado por Outokump. 
Como podemos apreciar, esta reacción no es espontánea, pero al agregar radiación ultravioleta, el $\Delta G$ (energía libre de Gibbs) cambia de signo y ocurre la ruptura hemolítica del peróxido de hidrógeno, formando así el radical hidroxilo, el cual posee un alto potencial de reducción $\left(E^{\circ}=2,8 \mathrm{v}\right)$. Es decir, que si bien es cierto el peróxido de hidrógeno es un agente oxidante, pero un agente oxidante más potente que este es el ion hidroxilo, debido a que tiene un potencial de reducción más alto que el del peróxido de hidrógeno y es el que va actuar en la fotooxidación del colorante.

Las soluciones del colorante rojo de synosol, son ácidas debido a que posee hidrógenos provenientes de un ácido carboxílico, como se puede apreciar en el espectro infrarrojo (señal a $3500 \mathrm{~cm}^{-1}$ ).

Gráfico 1. Espectro infrarrojo del rojo de synosol

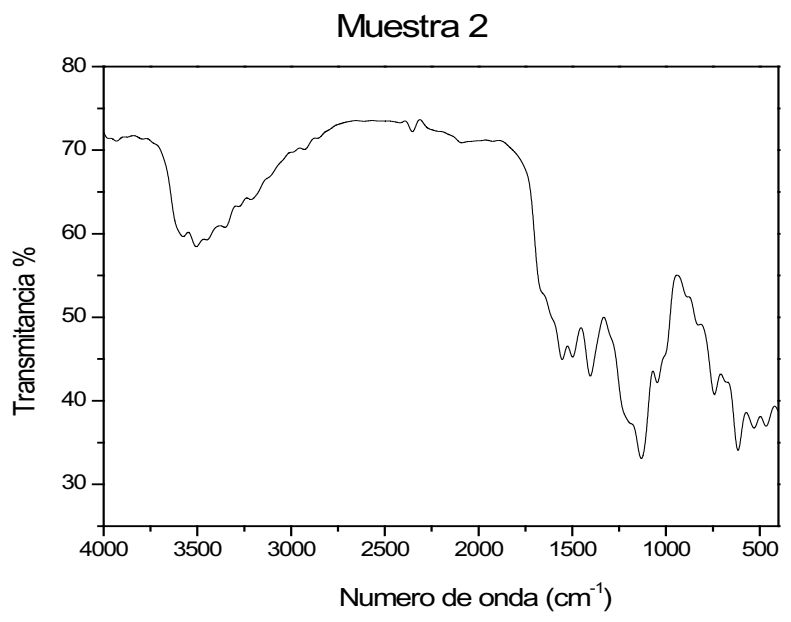

\section{Experimento 1:}

Se preparó una muestra sintética de 100 ppm de rojo de synosol, al cual se acondicionó a $\mathrm{pH} 6$, utilizando hidróxido de sodio $4 \mathrm{M}$. Se mantuvo una concentración constante de 100 ppm de dióxido de titanio y sólo se varió la concentración de peróxido de hidrógeno (agua oxigenada). Se expuso a luz ultravioleta por espacio de 1 y $2 \mathrm{~h}$. Los resultados se aprecian en el cuadro 2 y se ilustran en el Gráfico N. ${ }^{\circ}$ 2. La potencia de la lámpara UV es de $8 \mathrm{~W}$.

En el Gráfico N.$^{\circ} 2$ se aprecia una mayor disminución del color a $0,005 \%$ de peróxido de hidrógeno (agua oxigenada) y en cuanto a los tiempos de exposición de radiación UV, es más efectivo a un tiempo de exposición de 2 horas.

\section{Experimento 2:}

Con el dato de concentración de agua oxigenada de $0,005 \%$, obtenido del experimento anterior, esta se mantuvo constante y se preparó una muestra sintética de 100 ppm de rojo de synosol, al cual se acondicionó a pH 6, utilizando hidróxido de sodio $4 \mathrm{M}$. Sólo se varió la concentración de dióxido de titanio. Se expuso a luz ultravioleta por espacios de $1,1,5$ y $2 \mathrm{~h}$. Los resultados se aprecian en el cuadro 3 y se ilustran en el Gráfico N.$^{\circ} 3$. La potencia de la lámpara UV es de $8 \mathrm{~W}$.

De acuerdo al Gráfico N. ${ }^{\circ} 3$, se aprecia una mayor disminución del color a 0,005 \% de peróxido de hidrógeno (agua oxigenada) y a menores concentraciones del dióxido de titanio (50 ppm TiO2), el sistema de degradación de color se comporta mejor y el tiempo que registra mejores resultados corresponde a 2 horas de exposición a la radiación UV.

Cuadro 2

\begin{tabular}{ccc}
\hline $\mathrm{H}_{2} \mathrm{O}_{2}$ & \multicolumn{2}{c}{$\%$ color remanente } \\
\hline$\%$ & $1 \mathrm{~h}$ & $2 \mathrm{~h}$ \\
0,015 & 51,8 & 20,2 \\
0,013 & 39,6 & 14,4 \\
0,010 & 37,9 & 12,1 \\
0,008 & 38,7 & 14,6 \\
0,005 & 30,4 & 12,2 \\
\hline
\end{tabular}

Cuadro 3

\begin{tabular}{cccc}
\hline \multirow{2}{*}{$\begin{array}{c}\text { TiO2 } \\
\text { ppm }\end{array}$} & \multicolumn{3}{c}{ \% color remanente } \\
\cline { 2 - 4 } & $\mathbf{1 ~ h}$ & $\mathbf{1 , 5} \mathbf{h}$ & $\mathbf{2 ~ h}$ \\
\hline 150 & 44,1 & 23,9 & 8,8 \\
\hline 125 & 33,2 & 20,7 & 9,9 \\
\hline 100 & 37 & 14,6 & 3,1 \\
\hline 75 & 26,6 & 10,6 & 1,7 \\
\hline 50 & 24,9 & 8,8 & 0,2 \\
\hline
\end{tabular}


Gráfico 2. Disminución del color en función de la concentración de peróxido de hidrógeno (agua oxigenada)

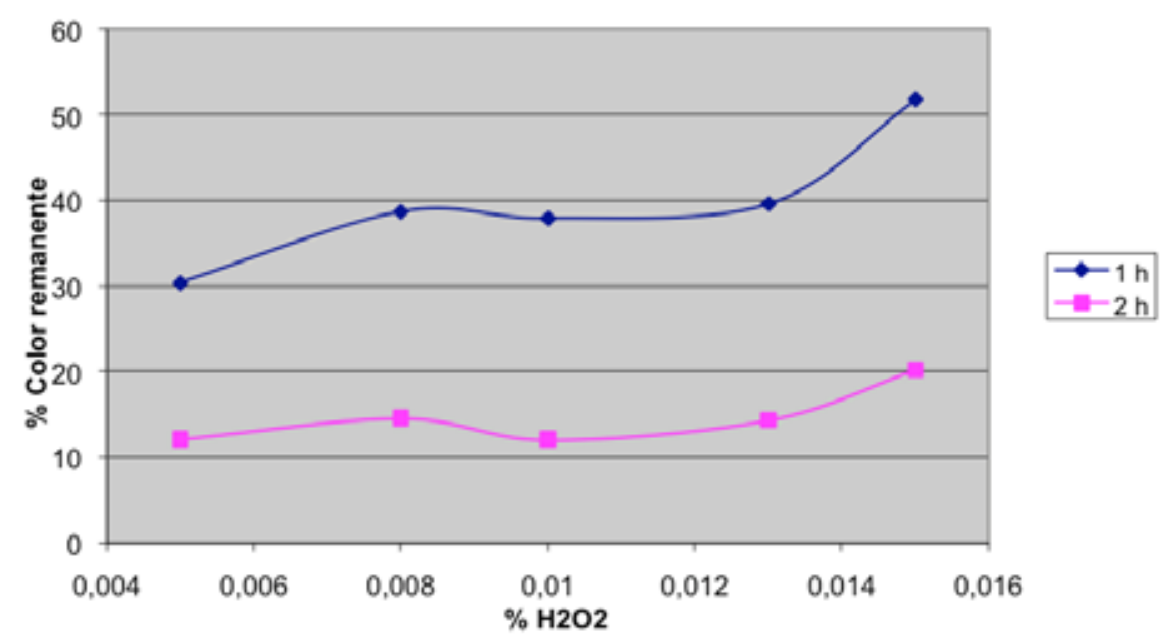

Gráfico 3. Disminución del color en función de la concentración de dióxido de titanio

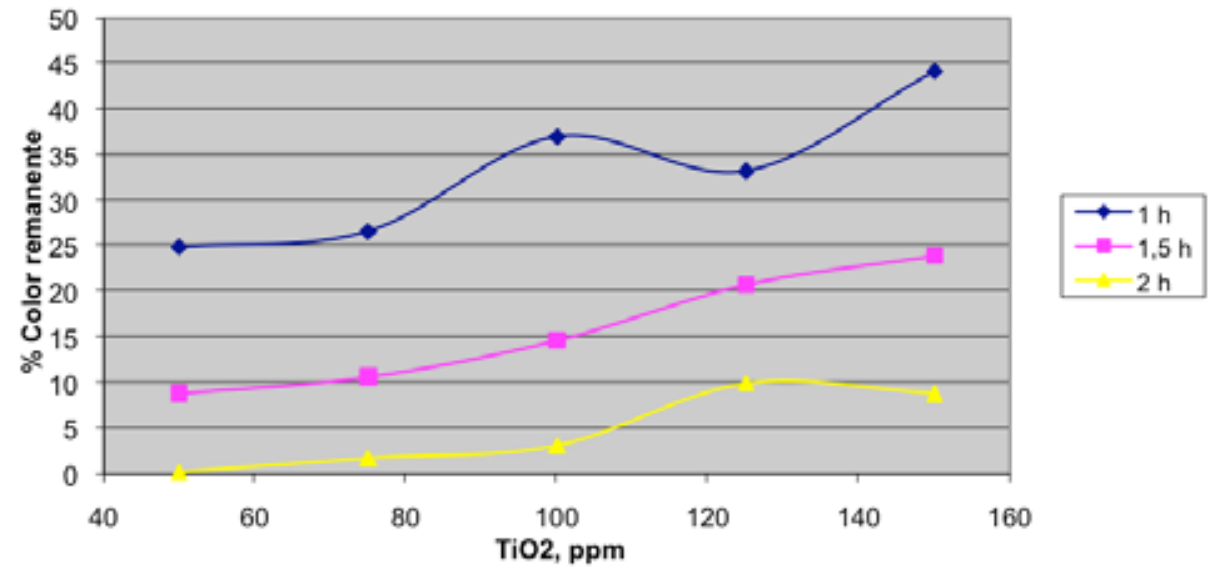

\section{DISCUSIÓN}

- En el Gráfico N. ${ }^{\circ}$ 2, se aprecia que a menores concentraciones de peróxido de hidrógeno (agua oxigenada), la pérdida de color del colorante es mayor. Ello se debe a que una menor concentración de agua oxigenada, genera una menor formación de radicales hidroxilo, y por ende la concentración de estos es pequeña, evitando así que reaccionen entre ellos, y se neutralicen antes de realizar su acción oxidativa sobre el colorante.
- En el Gráfico N. ${ }^{\circ}$ 2, también se aprecia que en el rango de concentraciones de 0,008 - 0,013 $\%$ de agua oxigenada, la pérdida de color es casi constante.

- En el Gráfico N. ${ }^{\circ} 3$, se aprecia que a menores concentraciones de dióxido de titanio la reacción presenta un mejor decoloramiento; ello podría deberse a que con menores concentraciones de dióxido de titanio, la formación del radical hidroxilo es menor, aumentando así su eficiencia en el proceso. 


\section{CONCLUSIONES}

- El tiempo óptimo de degradación del color es de 2 h y la concentración de dióxido de titanio para lograr dicho tiempo fue de $50 \mathrm{ppm}$.

- El dióxido de Titanio se confirma como fotocatalizador para la degradación fotocatalítica de efluentes textiles, con luz ultravioleta solar de baja energía

\section{REFERENCIAS BIBLIOGRÁFICAS}

[1] Chacón, Juan (2003). Mineralización de colorantes por foto Fenton y energía solar. Instituto mexicano de tecnología del agua.

[2] Crespi, M (2008) Industria Textil: ¿Depuración Biológica o Fisicoquímica? Boletín INTEXTAR. 1987, NQ 92.

[3] Garcés Fernando (2005). "Degradación de aguas residuales de la industria textil por medio de fotocatálisis. Revista lasallista de investigación. Vol 2 N. ${ }^{\circ} 1$.
[4] Mansilla et al. (2002). Tratamiento de residuos líquidos de la industria textil y celulosa. Consultado el 15-8-2011 en: http://www.cnea.gov.ar/ xxi/ambiental/cyted/20cap13.pdf

[5] Red CYTED VIII-G, Argentina. Texto colectivo. Consultado el 22-9-2011 en: http://www.psa.es/ webesp/projects/solarsafewater/documents/libro/00_Introduccion.pdf

[6] Rodríguez, Antonio et al. (2006) Tratamientos avanzados de aguas residuales industriales. Círculo de Innovación en Tecnologías Medioambientales y Energía (CITME). Universidad de Alcála. España

[7] Salas, G. (2003). Tratamiento fisico-químico de aguas residuales de la industria textil. Revista Peruana de Ing. Química. Vol 5 N..$^{\circ} 2$.

[8] Silva, Antonio (2005). Degradación fotocatalítica del Azo colorante Azul - 71 del efluente textil sobre uv/tio ${ }_{2}$. Anales de la Universidad Nacional Agraria de La Molina (UNALM). Lima. 